bers via the industrial workshops and the many initiatives of ACAPPI has continued so as to expand interest in, and support for, EPS. The Conference Committee has played an essential rôle in the contacts with the ESF and the CEC in relation to the ERC and Europhysics Conferences. The Physics and Society Committee has reported on the Europhysics Study Conference on "Induced Critical Conditions in the Atmosphere" held in Turin (September 1989) and on the Heraeus Seminar on "Balances in the Atmosphere and the Energy Problem" held in Bad Honnef (February 1990). The east - west meeting held in Amsterdam at EPS-8 was also an ACPS activity. The next initiatives be will be a seminar on nuclear safety and a Study Conference titled "Science Policy and Management".

Science and technology are inevitably conditioned by the society in which they are immersed. The rôle of scientific communities therefore acquires a significance extending beyond the simple question of providing scientific research and information describing its results. The EPS's achievements over 22 years go a long way towards fulfilling the aims of its founders. Cooperation among national societies and individual physicists in eastern and western countries is now the basis for a real European future for our community. We have to consider the development of this cooperation as the major task for the new EPS which, provided every "European physicist" does not refuse personal support, can become yet another example of a revived scientific culture.

\section{R.A. Ricci, EPS President}

\section{East-West Success}

Professor O.G. Folberth, Chairman of the task force set up after the General Meeting in Amsterdam last autumn to coordinate east-west initiatives, reports two notable successes. They go some way to meeting the urgent needs that were singled out in the 1990 report on how to help physicists in eastern Europe.

The first is an agreement with IBM to create several academic computer centres in eastern Europe, each with an IBM 3090 machine, that will be linked to the EARN computer network. Plans are well advanced in Poland, Hungary and Czechoslovakia where some sites have already been designated in collaboration with government officials. Discussions are in progress in Yugoslavia but more work has yet to be done in the remaining eastern countries.

The other good news is a donation from the WE Heraeus Foundation to pay for 59 subscriptions of Europhysics Letters for three years. The EPS Action Committee on Publications is meanwhile carrying out a survey to establish which journals are needed and by whom.

\title{
Physics and Physicists for Development
}

IGPD, the Interdivisional Group on Physics for Development, of the EPS organized a meeting titled "Physics and Physicists for Development" at the University of Twente, Enschede, The Netherlands on 10/11 September 1990. It was attended by speakers from countries ranging from the least to the most developed. In summarizing discussions it was unanimously agreed that physicists must use all available means to point out the importance of basic science for development, and especially stress that "long term progress is only possible if a percentage of government funds, and in particular of the money available from development assistance, is used for teaching the basic sciences and for carrying out research".

In short, the message was that a sustainable technological development, not to speak of technology transfer, is dependent upon an awareness by the general public of principles of physics and technology. These are obtainable only through a proper education in physics, with experimental activities for pupils and students during at least the last two years of high school and beyond.

The participants agreed unanimously to have the conclusions of the meeting distributed as a Declaration.

\section{DECLARATION}

1) Any country, "developing" as well industrialized, wanting to secure a stable society in which industrialisation, public health care, advanced agriculture and all other fields using applied sciences can flourish, inevitably needs a highly developed education and research programme in the basic sciences.

2) The essential rôle of basic science is not generally accepted by politicians and the public, especially in Third World countries where so many urgent needs are felt. It is often assumed that applied sciences are sufficient. This is not true. The development or the attempted direct transfer of technology should be preceded by a build up from basic knowledge. As physics is the fundamental science for the greater part of instrumentation and technology, there is special need for physics research and education with the emphasis on experimental activities.

3) Although fundamental research may not always be immediately feasible, the aim should be to include it as an essential feature in every country, as a means to sharpen analytic power and to raise the quality of education.

4) An enormous amount of talent is wasted, especially in the Third World, since it is not developed. Building an adequate education system should be a political priority in every country. As far as physics is concerned, creative teaching and experimentation is needed first and foremost: costly equipment is not the prime necessity, al-

\section{Max Born Medal and Prize}

The 1991 Max Born Medal and Prize awarded jointly by the Institute of Physics and the German Physical Society has been won by G.G. Lonzarich of the Cavendish Laboratory, Cambridge, for the development of techniques to study the De Haas-van Alphen effect. though it remains a significant problem as scope is widened.

5) Long term progress for sustained technological development is only possible if a percentage of government funds, in particularly monies from development assistance programmes, is used for teaching the basic sciences and carrying out research in these fields.

6) International cooperation is of the greatest importance, but it can only be successful if it is tailor-made. In-depth knowledge of local conditions, circumstances and culture is needed for those taking part in the development of an educational programme. An education system for the sciences leaning heavily on foreign experts may easily fail if this is not taken into account. The same also holds for research activities based entirely on expatriates and not properly anchored in the society.

7) The monitoring of cooperation projects is important for gaining an insight into the conditions for success and failure.

8) Given this background, we find that the world-wide community of universities, with a mission for all people, should see its duty as taking part in the development of adequate education systems and research activities, tailormade for different local conditions, to give talents latent in every country the opportunity to reach their full potential.

\section{E. Lillethun}

Chairman, IGPD

\section{Holweck Prize}

The winner of the 1991 Holweck Prize of the French Physical Society and the IOP is Professor A. Aspect of the Laboratoire de Spectroscopie Hertzienne de l'Ecole Normale Supérieure in Paris, for theoretical and experimental contributions to the study of ultra-cold atoms. 